\title{
Preeclampsia Selectively Impairs Endothelium-dependent Relaxation and Leads to Oscillatory Activity in Small Omental Arteries
}

\author{
Istenio F. Pascoal, ${ }^{\ddagger}$ Marshall D. Lindheimer, ${ }^{\star \star \S}$ Carol Nalbantian-Brandt, ${ }^{\ddagger}$ and Jason G. Umans ${ }^{\star \S}$ \\ ${ }^{*}$ Department of Medicine and ${ }^{\ddagger}$ Department of Obstetrics and Gynecology, and the ${ }^{\S}$ Committee on Clinical Pharmacology, Division of \\ Biological Sciences, University of Chicago, Chicago, Illinois 60637
}

\begin{abstract}
The vascular pathophysiology of preeclampsia, a hypertensive disorder unique to human pregnancy, has been postulated to be due to endothelial dysfunction, primarily manifest as deficient nitric oxide (NO) synthesis. We evaluated contraction ( $\mathrm{KCl}$ and arginine vasopressin [AVP]) and dilation (acetylcholine and bradykinin) in small resistance-size omental arteries obtained during surgery from women with preeclampsia, postulating that these vessels would exhibit augmented contraction and diminished endothelium-dependent relaxation, most likely due to decreased NO synthesis. For comparison, vessels were also obtained from normotensive gravidas, pregnant women with chronic hypertension, or with chronic hypertension and superimposed preeclampsia, as well as from premenopausal nonpregnant controls. Vessels of $\sim 200 \mu \mathrm{m}$ in internal diameter were studied in vitro using a Mulvany-Halpern myograph. Maximal contraction due to either $\mathrm{KCl}$ or AVP was significantly augmented in vessels from women with preeclampsia; these vessels all exhibited endothelium- and cyclooxygenase-dependent phasic oscillations while vessels from all other groups exhibited only tonic contractions. Acetylcholine and bradykinin both led to dose- and endothelium-dependent relaxation which was unaffected by inhibitors of NO synthesis. Responses to bradykinin were similar in vessels from normal pregnant and preeclamptic women while those to acetylcholine were absent in vessels from women with preeclampsia. These data suggest specific defects in resistance-artery endothelium from women with preeclampsia. (J. Clin. Invest. 1998. 101:464-470.) Key words: pregnancy • preeclampsia • endothelium • arteries $\bullet$ hypertension
\end{abstract}

\section{Introduction}

Preeclampsia is a potentially life-threatening multisystem disorder which presents with de novo hypertension and proteinuria in late pregnancy (1). Its vascular pathophysiology includes a striking reversal in the changes associated with normal gestation $(1,2)$. In normal pregnancy, systemic vasodilation

Address correspondence to Jason G. Umans, M.D., Ph.D., Section of Nephrology, University of Chicago, 5841 S. Maryland Avenue, MC5100, Chicago, IL 60637. FAX: 312-702-5818; E-mail: jumans@medicine. bsd.uchicago.edu

Received for publication 5 May 1997 and accepted in revised form 15 November 1997.

J. Clin. Invest.

(C) The American Society for Clinical Investigation, Inc. 0021-9738/98/01/0464/07 \$2.00

Volume 101, Number 2, January 1998, 464-470

http://www.jci.org exceeds the large increment in cardiac output, leading to decreased arterial BP; while preeclampsia is characterized by normal or decreased cardiac output but increased BP due to large increments in systemic vascular resistance. Also, vasculature of normotensive pregnant women is refractory to angiotensin II, but in preeclampsia vasopressor sensitivity to this peptide is increased, perhaps even before overt manifestation of disease (1).

The cause of preeclampsia is unknown. However, recent studies suggest that increments in BP may reflect endothelial dysfunction. First, there is morphological and biochemical evidence that preeclampsia is preceded and/or accompanied by subtle endothelial damage $(3,4)$. Second, there are observations suggesting that gestational vasodilation reflects increments in production of endothelial relaxing factors, primarily nitric oxide $(\mathrm{NO})^{1}(5-8)$. In this respect, NO synthase inhibition evokes hypertension and proteinuria in pregnant rats, when urinary nitrate-nitrite excretion, though depressed, is still equal to that of virgin animals (9). Thus, decreased elaboration of endogenous vasodilators (primarily $\mathrm{NO}$ ) has been proposed as a mechanism for the pathogenesis or perpetuation of hypertension in preeclampsia. Our studies were designed to compare contraction and dilation in small resistance-size omental arteries obtained from women with preeclampsia, postulating that these vessels would exhibit augmented contraction and diminished endothelium-dependent relaxation. We further hypothesized that the endothelial dysfunction would reflect decreased production of NO.

Our results do indeed demonstrate augmented contraction in vessels from women with preeclampsia, which failed to dilate when treated with acetylcholine (ACh), but not with another endothelium-dependent vasodilator. In addition, preeclampsia led to endothelium-dependent oscillatory activity in submaximally constricted vessels. Surprisingly, endotheliumdependent relaxation of these human omental vessels was NO independent. Thus, preeclampsia alters both contraction and relaxation in these vessels by selectively impairing endothelial function in a manner which does not depend on changes in the basal or vasodilator-stimulated release of an NO-containing endothelium-derived relaxing factor.

\section{Methods}

Subjects. Omental fat pad biopsies were performed during cesarian section or laparotomy in six groups of volunteers (Table I): group 1, normotensive primigravid women; group 2 , normotensive multigravi-

1. Abbreviations used in this paper: ACh, acetylcholine; AVP, arginine vasopressin; BK, bradykinin; HELLP syndrome, acronym for a disease variant which stands for Hemolysis, Elevated Liver enzymes Low Platelet count; INDO, indomethacin; NNLA, $N^{\mathrm{G}}$-nitro-L-arginine; NO, nitric oxide; PSS, physiological salt solution; SNP, sodium nitroprusside. 


\begin{tabular}{|c|c|c|c|c|c|}
\hline & Gestational age & SBP & DPB & Proteinuria & Fetal weight \\
\hline & $w k$ & $m m H g$ & $m m H g$ & + & $k g$ \\
\hline \multicolumn{6}{|l|}{ Nulliparous } \\
\hline Normotensive $(n=11$, age $=29 \pm 7)$ & $39 \pm 1$ & $118 \pm 11$ & $69 \pm 7$ & Negative & $3.3 \pm 0.7$ \\
\hline Preeclamptic $(n=11$, age $=24 \pm 6)$ & $33 \pm 4 *$ & $170 \pm 14 *$ & $103 \pm 10^{*}$ & $3 \pm 1$ & $2.1 \pm 1.2 *$ \\
\hline \multicolumn{6}{|l|}{ Parous } \\
\hline Normotensive $(n=11$, age $=29 \pm 5)$ & $38 \pm 2$ & $117 \pm 8$ & $71 \pm 5$ & Negative & $3.3 \pm 0.6$ \\
\hline Chronic hypertensive $(n=5$, age $=33 \pm 2)$ & $38 \pm 2$ & $134 \pm 4$ & $77 \pm 5$ & Negative & $3.5 \pm 0.8$ \\
\hline \multicolumn{6}{|l|}{ Chronic hypertensive + HELLP } \\
\hline$(n=4$, age $=32 \pm 4)$ & $32 \pm 2$ & $173 \pm 13$ & $105 \pm 5^{\ddagger}$ & $2 \pm 1$ & $1.8 \pm 1.0^{\ddagger}$ \\
\hline \multicolumn{6}{|l|}{ Control } \\
\hline Nonpregnant $(n=11$, age $=38 \pm 5)$ & & $126 \pm 8$ & $71 \pm 5$ & Negative & \\
\hline
\end{tabular}

*Preeclamptic versus nulliparous normotensive; ${ }^{\ddagger}$ Chronic hypertensive + HELLP versus chronic hypertensive $(P<0.05) . S B P$, Systolic blood pressure; $D B P$, diastolic blood pressure. Data are mean $\pm \mathrm{SD}$.

das; group 3, primigravidas with preeclampsia; group 4, pregnant women with chronic hypertension; group 5, pregnant women with chronic hypertension and superimposed preeclampsia; and group 6, nonpregnant normotensive premenopausal women. Preeclampsia was defined as de novo hypertension (diastolic BP $\geq 90 \mathrm{mmHg}$, Korotkoff phase V) appearing after gestational week 20, accompanied by appearance of proteinuria ( $\geq 300 \mathrm{mg} / 24 \mathrm{~h}$, or $\geq 2+$ by dipstick), and a serum uric acid $\geq 5.5 \mathrm{mg} / \mathrm{dl}$. Chronic hypertension was diagnosed by history or by documentation of elevated BP before midgestation. The diagnosis of preeclampsia superimposed on chronic hypertension was made when the patients developed evidence of the "HELLP" syndrome (an acronym for a disease variant which stands for Hemolysis, Elevated Liver enzymes Low Platelet count), manifested by evidence of microangiopathic hemolytic anemia (i.e., schistocytes on the blood smear), elevated transaminase and lactate dehydrogenase, and thrombocytopenia. Exclusions included other diseases known to alter vascular responsiveness, including diabetes mellitus, renal disease, collagen vascular disorders, infection, and also estimated operative blood loss $>800 \mathrm{ml}$ (10). The research protocol was approved by the University of Chicago's Institutional Review Board, and informed consent was obtained in writing from each participant.

Microvessel preparation and tension measurements. Methods were basically as described previously by ourselves and others $(11,12)$. Briefly, the omental specimen $(\sim 2-4 \mathrm{~g})$ was immediately placed in cold oxygenated physiological salt solution (PSS), consisting of 118.2 $\mathrm{mM} \mathrm{NaCl}, 24.8 \mathrm{mM} \mathrm{NaHCO}_{3}, 4.6 \mathrm{mM} \mathrm{KCl}, 1.2 \mathrm{mM} \mathrm{KH} \mathrm{PO}_{4}, 1.2 \mathrm{mM}$
$\mathrm{MgSO}_{4}, 2.5 \mathrm{mM} \mathrm{CaCl}_{2}$, and $10.0 \mathrm{mM}$ dextrose. A small artery $(\sim 200$ $\mu \mathrm{m}$ normalized internal diameter $\left[\mathrm{L}_{0}\right]$ ) was dissected into two $0.5-\mathrm{mm}$ rings, which were each mounted in a Mulvany-Halpern myograph (Living Systems Instrumentation, Burlington, VT) using 16- $\mu \mathrm{m}$ tungsten wires. The myograph bath contained PSS at $37^{\circ} \mathrm{C}$, bubbled with $5 \% \mathrm{CO}_{2}$ in $\mathrm{O}_{2}$ ( $\left.\mathrm{pH} 7.4\right)$. After $1 \mathrm{~h}$ of equilibration, including six bath changes, the artery underwent two conditioning stretches of $0.6 \mathrm{mN} /$ $\mathrm{mm}$. Passive tension-circumference characteristics were determined, each ring was set to an $\mathrm{L}_{0}$ which resulted in wall stress equivalent to that due to a transmural pressure of $100 \mathrm{mmHg}$ (Table II), and was then studied under isometric conditions. Preliminary experiments demonstrated maximal arginine vasopressin (AVP)-induced contraction, with preservation of endothelium-dependent relaxation, when resting vessel circumference was set in this manner.

Study design. The arteries were initially contracted twice in potassium-substituted PSS (KSS) in which $60 \mathrm{mM}$ of $\mathrm{NaCl}$ was replaced by $\mathrm{KCl}$. After washing and reequilibration in PSS, a cumulative doseresponse curve was constructed for AVP (0.1-100 nM)-induced contraction. Endothelium-dependent relaxation by either ACh (0.01-10 $\mu \mathrm{M})$ or bradykinin (BK, 0.01-10 $\mu \mathrm{M})$ was then assessed in vessels which were submaximally preconstricted with AVP and this procedure subsequently repeated in the presence of $N^{\mathrm{G}}$-nitro-L-arginine (NNLA, $100 \mu \mathrm{M}$ ) and/or indomethacin (INDO, $10 \mu \mathrm{M}$ ). Responses after NNLA $(100 \mu \mathrm{M})$ were confirmed by observing similar effects with either NNLA $(1 \mathrm{mM})$ or methylene blue $(10 \mu \mathrm{M})$; similarly, meclofenamate $(10 \mu \mathrm{M})$ yielded responses similar to those observed with INDO. As well, time controls demonstrated reproducibility of

Table II. Vessel Dimensions and Contractile Responses

\begin{tabular}{|c|c|c|c|c|}
\hline & $\mathrm{KCl}(60 \mathrm{mM})$ & AVP $(100 \mathrm{nM})$ & $\operatorname{AVP} \operatorname{ED}_{50}(95 \% \mathrm{CI})$ & $\mathrm{L}_{\mathrm{o}}$ \\
\hline & $m N / m m$ & $m N / m m$ & $n M$ & $\mu m$ \\
\hline \multicolumn{5}{|l|}{ Nulliparous } \\
\hline Normotensive $(n=11)$ & $2.1 \pm 1.1$ & $3.1 \pm 1.2$ & $0.78(0.54-1.12)$ & $197 \pm 34$ \\
\hline Preeclamptic $(n=11)$ & $3.3 \pm 1.1 *$ & $4.5 \pm 1.2^{*}$ & $0.85(0.49-1.49)$ & $197 \pm 39$ \\
\hline \multicolumn{5}{|l|}{ Parous } \\
\hline Normotensive $(n=10)$ & $2.4 \pm 1.1$ & $3.1 \pm 1.3$ & $0.93(0.55-1.57)$ & $212 \pm 34$ \\
\hline Chronic hypertensive $(n=5)$ & $2.7 \pm 0.7$ & $3.2 \pm 0.6$ & $0.93(0.59-1.46)$ & $181 \pm 19$ \\
\hline Chronic hypertensive $+\operatorname{HELLP}(n=4)$ & $3.4 \pm 0.4$ & $5.0 \pm 0.6^{\ddagger}$ & $0.76(0.33-1.75)$ & $217 \pm 71$ \\
\hline \multicolumn{5}{|l|}{ Control } \\
\hline Nonpregnant $(n=11)$ & $2.7 \pm 1.1$ & $3.2 \pm 1.2$ & $0.77(0.40-1.40)$ & $209 \pm 55$ \\
\hline
\end{tabular}

*Preeclamptic versus nulliparous normotensive; ${ }^{\ddagger}$ Chronic hypertensive + HELLP versus chronic hypertensive $(P<0.05)$. Data are mean \pm SD. 
relaxation in the absence of added inhibitors and stability of contraction in the absence of added vasodilator. At the end of each experiment, endothelium-independent, cGMP-mediated relaxation was assessed with the exogenous NO-donor sodium nitroprusside (SNP, 10 $\mu \mathrm{M}$ ); we have noted previously that SNP-induced relaxation of these omental vessels is inhibited by methylene blue. In addition, some rings were mechanically denuded of their endothelium by passing a human hair of appropriate diameter through the vessel lumen, and the relaxation protocols were repeated.

Chemicals. ACh chloride, AVP, BK, SNP, INDO, and NNLA were obtained from Sigma Chemical (St. Louis, MO). Stock solutions of reagents were prepared fresh daily in distilled water and diluted serially.

Data analysis. Sigmoid dose-response curves were individually fit to a log-logistic equation; maximal responses are presented as mean $\pm \mathrm{SD}$ and potency as $-\log \mathrm{EC}_{50}$. Data from multiple rings from the same subject were averaged; $n$ refers to the number of subjects. Group data were compared by paired and unpaired $t$ tests, with correction for multiple comparisons, as appropriate. $P$ values $<0.05$ were considered significant.

\section{Results}

Patient demographics. The vessels of 53 women were studied including 11 nulliparous normotensive gravidas, 11 parous normotensive gravidas, 11 nulliparous preeclamptic women, 5 pregnant women with chronic hypertension, 4 chronically hypertensive women with superimposed preeclampsia, and 11 nonpregnant subjects (Table I). The nulliparous normotensive pregnant women served as controls for the nulliparous preeclamptic women, while the parous normotensive gravidas were matched to the women with chronic hypertension, all of whom were multiparous. Women with preeclampsia all manifested proteinuric hypertension, by definition, and tended to deliver low birthweight babies prematurely. Most of the women with chronic hypertension had adequately controlled BP.

Contraction studies. Maximal contractions, whether induced by KSS or AVP, were augmented in arteries from nulliparous preeclamptic women compared with those from normotensive gravidas (Table II). Contraction was also enhanced

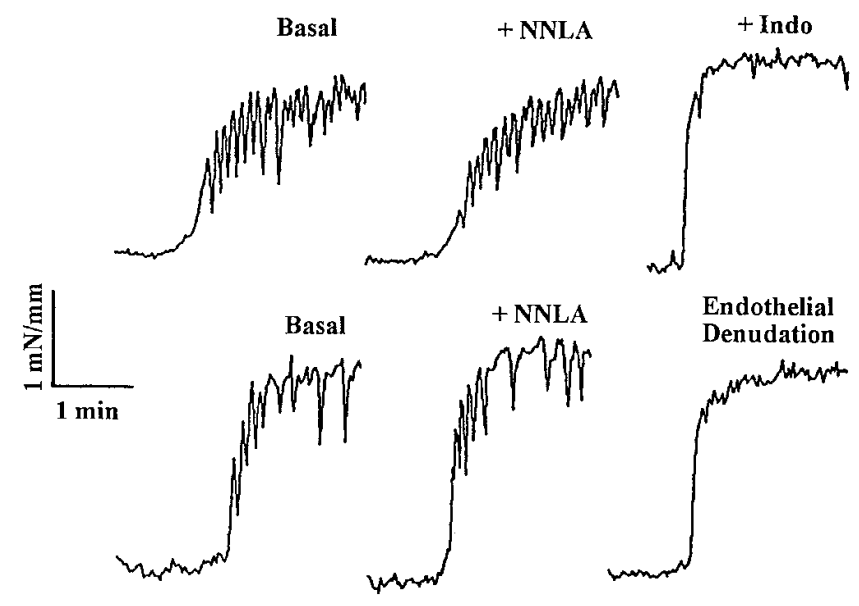

Figure 2. Six traces (force versus time) are shown for two vessels from preeclamptic patients. The two left-hand traces show oscillatory activity after preconstriction with a single dose of AVP. The two middle traces show that pretreatment with the NO synthase inhibitor NNLA $\left(10^{-4} \mathrm{M}\right)$ failed to alter these oscillations. The top right-hand trace shows that pretreatment with INDO $\left(10^{-5} \mathrm{M}\right)$ abolished the oscillations. Endothelial denudation (bottom right-hand trace) similarly abolished oscillations, again resulting in contraction which did not differ from that observed at the peak of the previously observed phasic activity.

in patients with superimposed HELLP syndrome when compared with pregnant chronic hypertensive patients, such that it did not differ from values in preeclamptic nulliparas. By contrast, there were no differences in maximal contraction when comparing vessels from any group without a diagnosis of either pure or superimposed preeclampsia, whether or not they were pregnant, parous, or had a history of hypertension. Vascular sensitivity to AVP, expressed as $E_{50} \mathrm{~s}$, did not differ among groups (Table II).

Vessels from preeclamptic women, or from those with

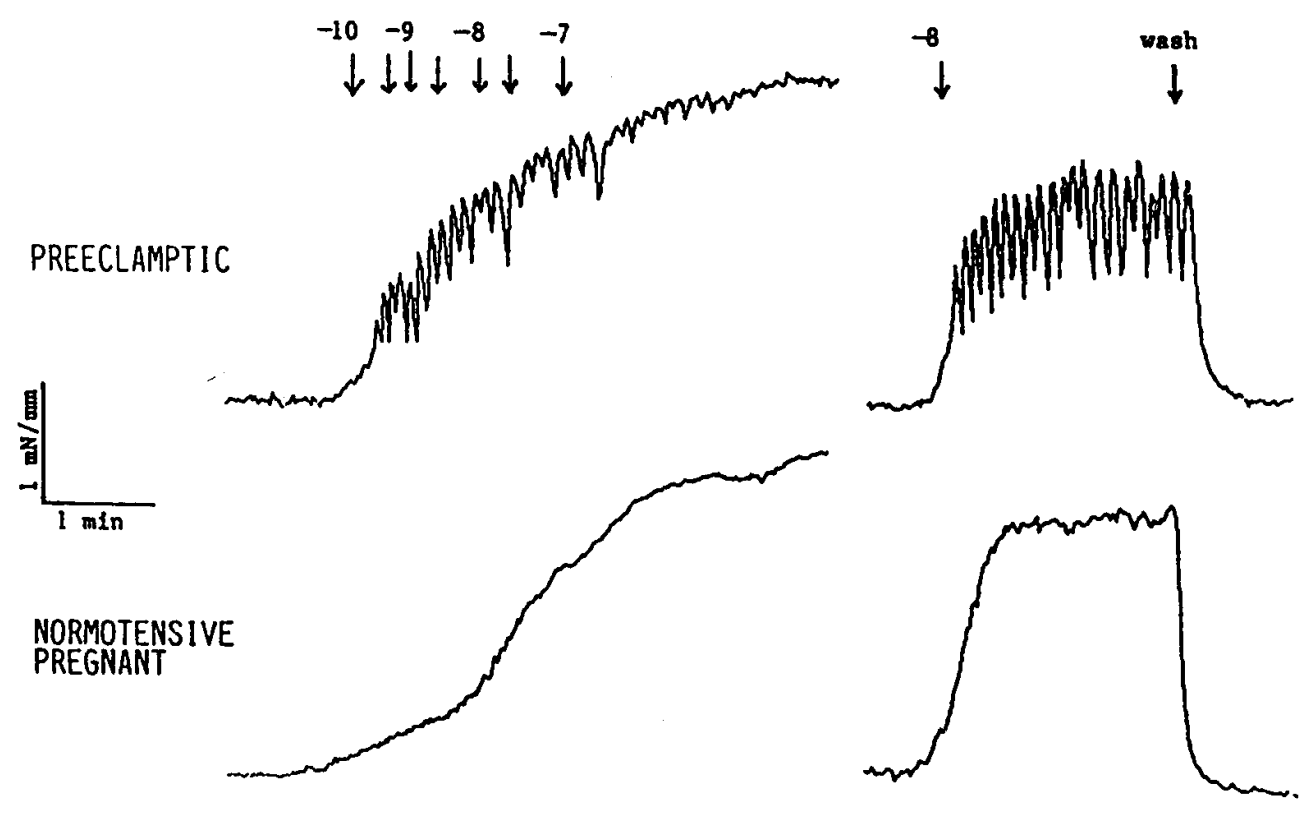

Figure 1. Representative original traces of force versus time for vessels from a preeclamptic woman (top traces) and from a normotensive gravida (bottom traces). Arrows denote the cumulative addition of AVP ( $\log$ concentration, $\mathrm{M}$ ). In the righthand traces, AVP was added at only a single, submaximally effective concentration of $10^{-8} \mathrm{M}$ At all intermediately effective doses, AVP led to phasic oscillatory activity in the vessel from the preeclamptic woman; only tonic responses were observed in the vessel from the normal gravida. 


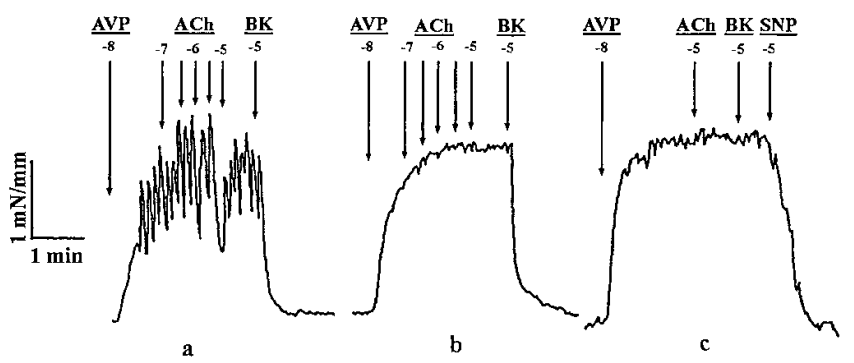

Figure 3. Three traces (force versus time) are shown from a single vessel from a preeclamptic patient. Trace $a$ shows AVP preconstriction leading to oscillatory activity, without apparent relaxation after graded addition of ACh. BK $\left(10^{-5} \mathrm{M}\right)$ led to complete relaxation and cessation of oscillations. Trace $b$, after addition of INDO $\left(10^{-5} \mathrm{M}\right)$ to abolish oscillations, again demonstrates no relaxation after $\mathrm{ACh}$ but complete relaxation after BK. Trace $c$, after denudation of the endothelium, shows loss of (endothelium-dependent) BK-induced relaxation, with preservation of relaxation induced by the nitrovasodilator SNP.

HELLP syndrome, all exhibited characteristic oscillations after intermediate doses of AVP, with tonic contractions observed only at maximal doses (Fig. 1). By contrast, vessels from normotensive gravidas exhibited only tonic contractions at all AVP doses. Oscillations in arteries from women with preeclampsia were abolished by either endothelial denudation or by cyclooxygenase inhibition with either INDO or meclofenamate, but not by NO synthase inhibition (Fig. 2). In each case, cyclooxygenase inhibition led to tonic contractions similar to those observed at the peak of oscillations, suggesting that the oscillations represented phasic relaxation.

Dilation studies. Vessels from normotensive pregnant women relaxed completely and in a strictly endothelium-dependent manner (i.e., vessels failed to relax after mechanical denudation of endothelium), in response to either ACh or BK, as has been shown previously (11). By contrast, ACh was without effect in vessels from preeclamptic women (Fig. $3 a$ ), an effect which could be observed more clearly when preeclamptic oscillations were abolished by addition of INDO (Fig. $3 b$ ). Unlike $\mathrm{ACh}, \mathrm{BK}$ relaxed vessels from preeclamptic women in the absence or presence of INDO; its effect was only abolished after removal of the endothelium (Fig. $3 c$ ). Indeed, complete dose-response curves showed that preeclampsia was without significant effect on BK-induced relaxation, while it abolished the response due to ACh (Fig. 4). Vessels from patients with superimposed preeclampsia exhibited the same selective relaxation defect to $\mathrm{ACh}$ as vessels from preeclamptic nulliparas. By contrast, vessels from all other groups relaxed completely in response to either agent.

NO synthase inhibition. NNLA was used both to reveal contributions of basal NO synthesis to modulating vasoconstrictor responses and to determine the role of NO in the doseand endothelium-dependent relaxations induced by either ACh or BK. Indeed, NNLA $(0.1 \mathrm{mM})$ led to small increments in response to submaximal doses of AVP, implying contributions of basal NO synthesis to modulate vasoconstriction in these vessels. Comparable contractile augmentation was observed in vessels from normal gravidas and preeclamptic women (normal $-\mathrm{NNLA} /+\mathrm{NNLA}$ : $2.05 \pm 1.20$ vs. $2.20 \pm 1.28$ $\mathrm{mN} / \mathrm{mm}, n=16, P=0.006$; preeclamptic -NNLA/+NNLA: $2.36 \pm 1.09$ vs. $2.91 \pm 1.28 \mathrm{mN} / \mathrm{mm}, n=14, P=0.07)$. By contrast, NNLA failed to alter dose-dependent ACh- or BKinduced relaxation in arteries from any group (Fig. 5). Cyclooxygenase inhibition (data not shown) was similarly without effect on agonist-induced vasodilation.

\section{Discussion}

Preeclampsia is peculiar to human pregnancy, as no animal models completely mimic its protean manifestations. Therefore, we focused on human omental vessels small enough to contribute to the regulation of systemic vascular resistance. We found several abnormalities in small artery function, which may explain the hypertension associated with preeclampsia. First, in vitro contractility was augmented. These vessels also displaying unique phasic oscillations during submaximal contraction, which appeared to depend on an endothelial vasodilator prostanoid. Protocols to explore agonist-stimulated vasodilation were equally revealing. ACh and BK each relaxed arteries from normotensive gravidas in a dose-dependent, strictly endothelium-dependent, yet NO-independent man-
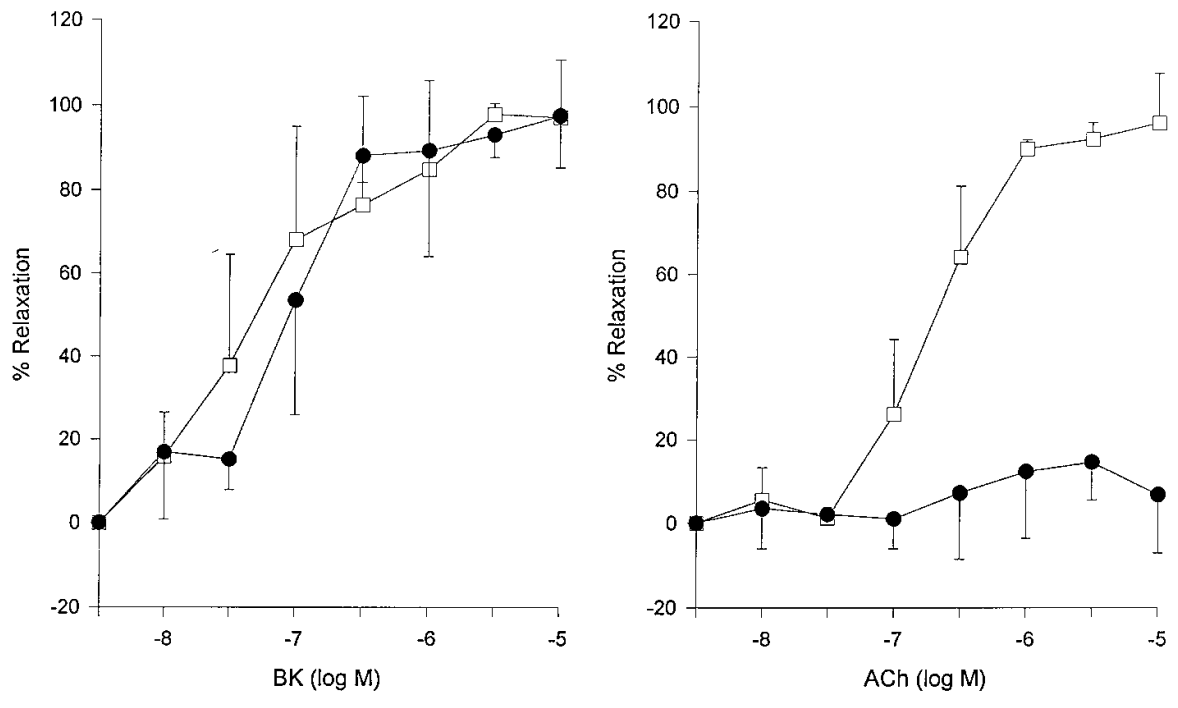

Figure 4. Dose-response curves for percent relaxation versus log concentration of BK (left) or ACh (right). Data from normal gravidas (open squares) and preeclamptic women (closed circles) are shown $( \pm \mathrm{SD})$. Dose-dependent BK-induced relaxation did not differ between groups. ACh-induced relaxation was selectively ablated in vessels from preeclamptic women. 

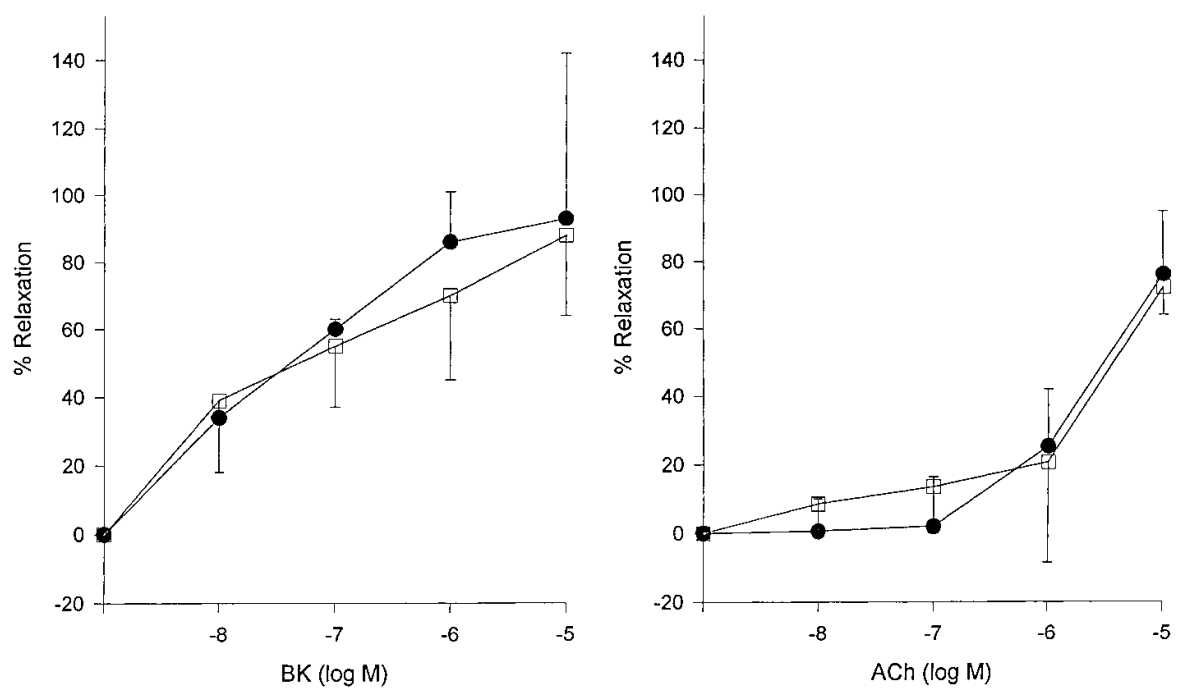

Figure 5. Dose-response curves for percent relaxation versus log concentration of BK (left) or ACh (right). Data $( \pm \mathrm{SD})$ are all from normal gravidas in the absence (open squares) or presence (closed circles) of the NO synthase inhibitor NNLA $\left(10^{-4} \mathrm{M}\right)$. Dose-dependent due to either endothelium dependent vasodilator was not altered significantly in the presence of NNLA. Similar results were obtained for BK-induced relaxation of vessels from preeclamptic women (not shown). ner. However, ACh failed to dilate the arteries from preeclamptic women, while the response of these vessels to BK remained intact. These data do not support views that gestational vasodilation is due primarily to increases in basal and vasodilator-stimulated release of $\mathrm{NO}$, or that vasoconstriction in preeclampsia reflects deficiencies in production of this factor.

Contractile properties of human omental vessels. Maximal contraction, induced by either potassium or AVP, was increased in preeclampsia, while reactivity in vessels from pregnant patients with chronic hypertension was unaltered, unless superimposed preeclampsia occurred. Our observation that preeclampsia augmented maximal contraction without a change in agonist potency differs with some reports in omental and subcutaneous resistance sized arteries $(13,14)$, and larger omental artery segments (15), but is in accord with one report using human inferior epigastric artery (16). Our findings are also consistent with observations that the media thickness/lumen diameter ratio of these vessels is increased in preeclampsia (13), leading to increased force generation when comparing vessels of similar caliber.

Oscillatory activity during submaximal contraction with AVP was another in vitro characteristic of vessels from women with preeclampsia but not those from nonpregnant women, normotensive gravidas, or chronic hypertensive gravidas; the latter vessels exhibited only tonic contractions. Since endothelial denudation or cyclooxygenase inhibition led to tonic contractions similar in magnitude to the peak of oscillatory tension, we concluded that these were phasic relaxations due to the action of an endothelium-derived prostanoid vasodilator. Since similar oscillations were not noted in subcutaneous resistance vessels from preeclamptic women (14), our observation may represent an example of regional vascular heterogeneity.

Aalkjaer et al. noted oscillations previously, but in vessels from both preeclamptic (14/18) and normotensive (3/15) gravidas (13). While such observations could represent pathophysiological phenomena, they may have also included artifact, since assessment of endothelial integrity was not a focus of protocols at that time. More importantly, most of their preeclamptic subjects were multigravidas, a group in which the clinical diagnosis of preeclampsia, even by strict criteria, is often incorrect (17). Our observations extend theirs (13) by es- tablishing that the oscillations are not artifactual, require an intact endothelium, appear unique to preeclampsia, and depend on cyclooxygenase. In these respects, a preliminary report (18) that rat mesenteric microvessels incubated in conditioned medium from hypoxic human trophoblast to mimic the placental hypoperfusion thought to eventuate in preeclampsia $(1,19)$ demonstrated not only increased vasoconstriction, but also exaggerated vasomotion (i.e., oscillations) is intriguing.

Oscillatory activity of myograph-mounted vessels has been observed in other experimental preparations. Strips from hamster aorta or rat small mesenteric vessels often display oscillations, which are exaggerated in some models of hypertension and depend on the continuous release of endothelium-derived NO, as the phasic activity can be ablated by endothelial denudation or NO inhibition, and then restored by exogenous nitrovasodilators or cGMP analogues (20-22). Such data, along with rhythmic variation in smooth muscle membrane potential (21), imply NO-mediated endothelial facilitation of spontaneous phasic activity arising in smooth muscle. Oscillations of both membrane potential and force were ablated by AChinduced hyperpolarization and vasorelaxation (21) and diminished coincident with hyperpolarization during normotensive rat pregnancy (23), suggesting inhibition by the hyperpolarizing vasodilator in these vessels (24). An analogous model, depending on facilitation by endothelial prostanoids, may explain our results.

The physiologic significance of vascular oscillations in resistance vessels remains unclear, with uncertainty as to whether they serve to increase or decrease vascular resistance and whether they may be linked in a causal way to hypertension (25), as opposed to representing a failed compensatory response to elevations in arterial pressure. Likewise, we do not know if these pathologic oscillations precede hypertension in women with preeclampsia.

Dilation in human omental vessels. Our postulate that altered NO synthesis would have central roles in mediating vascular changes in pregnancy (vasodilation) and preeclampsia (vasoconstriction) was based on various lines of evidence. Levels of NO metabolites and cGMP are increased (5) and NO has been implicated as the mediator of renal vasodilation and hyperfiltration in rat pregnancy (6). NO synthase inhibition se- 
lectively increases vascular resistance in the hand of human gravidas (8), inhibits ACh-induced relaxation of mesenteric microvessels in pregnant rats (7), and provokes proteinuric hypertension in this species (9). Yet, we found no role for NO in mediating ACh or BK effects in human omental vessels and no defect of basal NO synthesis in preeclampsia. Along with evidence that the augmented NO synthesis in rat pregnancy may not be of vascular origin (26), our data have led us to reevaluate mechanisms pertinent to human gestational vasodilation.

The role of hyperpolarizing vasodilators acting at $\mathrm{Ca}^{2+}$ activated $\mathrm{K}$ channels may dwarf that played by NO or cGMP in mediating endothelium-dependent relaxation of many rat arteries $(7,11,19,24,27,28)$. ACh-induced relaxation of human subcutaneous small arteries likewise depends more on a $\mathrm{K}$ conductance-mediated hyperpolarizing vasodilator than on NO (28) and maximal ACh- or BK-induced relaxations in human omental microvessels depend on a CTX-insensitive $\mathrm{K}$ channel, appearing glyburide-sensitive in some preparations (11). We extend these findings by showing that NO synthase inhibition fails to shift $\mathrm{ACh}$ or $\mathrm{BK}$ dose-response curves in normal gravidas. Importantly, our protocols were conducted in the absence of flow, a major stimulus for endothelial NO synthesis in vivo, suggesting caution in mechanistic comparison of our results with those from whole animal or perfused vessel systems.

Pregnancy augments endothelium-dependent relaxation of some rat vessel preparations, but not others $(7,29,30)$, perhaps by increasing sensitivity to flow or by favoring contributions of NO $(7,31,32)$. Studies of human resistance vessels in vitro are more limited; they suggest increased shear stress responsivity and contributions by a novel hyperpolarizing vasodilator, but no augmentation of ACh or BK effect $(11,33)$. There are no data regarding mechanisms which might effect these gestational changes or account for species differences.

BK-induced relaxation was well-preserved in vessels from preeclamptic women, though ACh failed to relax these arteries. This striking difference contrasts with results described in subcutaneous resistance vessels $(14,34)$, but is in accord with reports that flow-induced vasodilation is completely lost in these latter arteries from preeclamptic women (33) and that ACh-induced relaxation is abolished when these vessels are perfused with human syncytiotrophoblast microvillus membrane vesicles (35). Our observation of preserved endothelium-dependent BK responses and endothelium-independent SNP-induced relaxations suggests a specific endothelial target in preeclampsia, more likely a defect of muscarinic signal transduction than of endothelial receptors or relaxing factors, per se. Indeed, there is an informative precedent for this selective loss of endothelium-dependent relaxation to specific agonists, suggesting defects at the level of G protein-mediated signal transduction, vide infra.

Impaired endothelium-dependent relaxation to ACh has also been observed in conduit artery preparations from diabetic animals. In such preparations, defective endotheliumdependent relaxation might be due to glucose shunting through the polyol pathway leading to aldose-reductase stimulation (36) or to protein kinase C-mediated downregulation of NO synthase activity consequent to hyperglycemic diacylglycerol synthesis (37), although neither of these suggested mechanisms would predict selective defects in the response to different endothelium-dependent vasodilators. More recently, observation of selective deficits in ACh-, but not BK-induced relaxation of small mesenteric arteries from diabetic rats (38), led to the hypothesis of a mechanism based on protein kinase C-mediated phosphorylation of $\mathrm{G}_{\mathrm{i}}$ proteins (39). Likewise, both balloon injury and experimental atherosclerosis also lead to selective defects in agonist-induced endothelium-dependent vasodilation (40). Human hypercholesterolemia also results in impaired ACh- but not BK-induced forearm vasodilation in vivo (41). Taken together with our results in preeclampsia, these findings may all be consistent with a final effector pathway that interferes with $\mathrm{G}_{\mathrm{i}}$-mediated signal transduction and therefore would have the greatest effect on those agents or physical forces which seem to depend importantly on this pathway (42).

In summary, we found augmented contraction, exaggerated vasomotion, and selective ablation of ACh-induced relaxation in omental resistance arteries from women with preeclampsia. These effects all appear due to selective endothelial cell dysfunction. If such a vascular abnormality were to occur in vivo, it could represent an effector mechanism leading to the hypertension and pathologic vasospasm which characterize this uniquely human disorder.

\section{Acknowledgments}

The authors acknowledge Professor Atef Moawad for helpful discussions, support, and assistance in clinical aspects of this study.

I.F. Pascoal was a recipient of a postdoctoral fellowship from the Conselho Nacional de Desenvolvimento Cientifico e Tecnologico, Brazil (201635/92-1). J.G. Umans was a recipient of a career development award in clinical pharmacology from the Pharmaceutical Research and Manufacturers of America Foundation. This work was supported, in part, by grants from the American Heart Association of Metropolitan Chicago, Baxter Healthcare, the National Institutes of Health (HD-31939, HL-48302), and the Mother's Aid Research Fund of Chicago Lying-In Hospital.

\section{References}

1. August, P., and M.D. Lindheimer. 1995. Pathophysiology of preeclampsia. In Hypertension: Pathophysiology, Diagnosis, and Management. 2nd ed. J.H. Laragh and B.M. Brenner, editors. Raven Press Ltd., New York. 24072426.

2. Poppas, A., S.G. Shroff, C.E. Korcarz, J.U. Hibbard, D.S. Berger, M.D. Lindheimer, and R.M. Lang. 1997. Serial assessment of the cardiovascular system in normal pregnancy: role of arterial compliance and pulsatile arterial load. Circulation. 95:2407-2415.

3. Roberts, J.M., R.N. Taylor, and A. Goldfein. 1991. Clinical and biochemical evidence of endothelial cell dysfunction in the pregnancy syndrome preeclampsia. Am. J. Hypertens. 4:700-708.

4. Haller, H., E.-M. Ziegler, V. Hormuth, M. Drab, J. Eichhorn, Z. Nagy, A. Busjahn, K. Vetter, and F.C. Luft. 1997. Endothelial adhesion molecules and leukocyte integrins in preeclamptic patients. Hypertension. 29:291-296.

5. Conrad, K.P., G.M. Joffe, H. Kruszyna, R. Kruszyna, L.G. Rochelle, R.P. Smith, J.E. Chavez, and M.D. Mosher. 1993. Identification of increased nitric oxide biosynthesis during pregnancy in rats. FASEB (Fed. Am. Soc. Exp. Biol.) J. 7:566-571.

6. Danielson, L.A., and K.P. Conrad. 1995. Nitric oxide mediates renal vasodilation and hyperfiltration during pregnancy in chronically instrumented conscious rats. J. Clin. Invest. 96:482-490.

7. Pascoal, I.F., M.D. Lindheimer, C. Nalbantian-Brandt, and J.G. Umans. 1995. Mechanisms of acetylcholine-induced relaxation in mesenteric microvessels from pregnant rats. Am. J. Physiol. 269:H1899-H1904.

8. Williams, D.J., P.J.T. Vallance, G.H. Neild, J.A.D. Spencer, and F.J. Imms. 1997. Nitric oxide-mediated vasodilation in human pregnancy. Am. J. Physiol. 272:H748-H752.

9. Baylis, C., and K. Engels. 1992. Adverse interactions between pregnancy and a new model of systemic hypertension produced by chronic blockade of endothelial derived relaxing factor in the rat. Clin. Exp. Hypertens. B11:117-129.

10. Nelson, S.H., and M.S. Suresh. 1992. Lack of reactivity of uterine arter- 
ies from patients with obstetric hemorrhage. Am. J. Obstet. Gynecol. 166:14361443.

11. Pascoal, I.F., and J.G. Umans. 1996. Effect of pregnancy on mechanisms of relaxation in human omental microvessels. Hypertension. 28:183-187.

12. Aalkjaer, C., and M.J. Mulvany. 1981. Functional and morphological properties of human omental resistance arteries. Blood Vessels. 18:233-244.

13. Aalkjaer, C., H. Danielsen, P. Johannesen, E.B. Petersen, A. Rasmussen, and M.J. Mulvany. 1985. Abnormal vascular function and morphology in preeclampsia: a study of isolated resistance vessels. Clin. Sci. 69:477-482.

14. McCarthy, A.L., R.G. Woolfson, S.K. Raju, and L. Poston. 1993. Abnormal endothelial cell function of resistance arteries from women with preeclampsia. Am. J. Obstet. Gynecol. 168:1323-1330.

15. Belfort, M.A., G.R. Saade, M. Suresh, W. Kramer, and Y.P. Vedernikov. 1996. Effects of selected vasoconstrictor agonists on isolated omental artery from premenopausal nonpregnant women and from normal and preeclamptic pregnant women. Am J. Obstet. Gynecol. 174:687-693.

16. Ebeigbe, A.B., and M. Cabanie. 1991. In vitro vascular effects of cicletanine in pregnancy-induced hypertension. Br. J. Pharmacol. 103:1992-1996.

17. Fisher, K.A., A. Luger, B.H. Spargo, and M.D. Lindheimer. 1981. Hypertension in pregnancy: clinical-pathological correlations and remote prognosis. Medicine. 60:267-276.

18. Gratton, R.J., R.E. Gandley, J.F. McCarthy, and M.K. McLaughlin. 1996. Reduced vasomotion may contribute to the decrease in vascular resistance during pregnancy. J. Soc. Gynecol. Invest. 3:99a. (Abstr.)

19. Conrad, K.P., and D.F. Benyo. 1997. Placental cytokines and the pathogenesis of preeclampsia. Am. J. Reprod. Immunol. 37:240-249.

20. Jackson, W.F., A. Mulsch, and R. Busse. 1991. Rhythmic smooth muscle activity in hamster aortas is mediated by continuous release of nitric oxide from the endothelium. Am. J. Physiol. 260:H248-H253.

21. Gustafson, H. 1993. Vasomotion and underlying mechanisms in small arteries: an in vitro study of rat blood vessels. Acta Physiol. Scand. 614(Suppl.): $1-44$.

22. Lamb, F.S., and R.C. Webb. 1989. Potassium conductance and oscillatory contractions in tail arteries from genetically hypertensive rats. J. Hypertens. 7:457-463.

23. Meyer, M.C., J.E. Brayden, and M.K. McLaughlin. 1993. Characteristics of vascular smooth muscle in maternal resistance circulation during pregnancy in the rat. Am. J. Obstet. Gynecol. 169:1510-1516.

24. Hwa, J.J., L. Ghibaudi, P. Williams, and M. Chatterjee. 1994. Comparison of acetylcholine-dependent relaxation in large and small arteries of rat mesenteric vascular bed. Am. J. Physiol. 266:H952-H958.

25. Mulvany, M.J. 1988. Possible role of vascular oscillatory activity in the development of high blood pressure in spontaneously hypertensive rats. J. Cardiovasc. Pharmacol. 12:S16-S20.

26. Suto, T., A. Lateef, and C. Baylis. 1997. Effect of different nitric oxide synthesis inhibitors on nitric oxide production and blood pressure in pregnancy. Hypertens. Pregnancy. 16:68a. (Abstr.)

27. Cowan, C.L., J.J. Palacino, S. Najibi, and R.A. Cohen. 1993. Potassium channel-mediated relaxation to acetylcholine in rabbit arteries. J. Pharmacol.
Exp. Ther. 266:1482-1489.

28. Deng, L.Y., J.S. Li, and E.L. Schiffrin. 1995. Endothelium-dependent relaxation of small arteries from essential hypertensive patients: mechanisms and comparison with normotensive subjects and with responses of vessels from spontaneously hypertensive rats. Clin. Sci. 88:611-622.

29. Whittemore, S.L., M.K. McLaughlin, S.T. Davidge, and K.P. Conrad. 1994. Effect of pregnancy on vascular cGMP production and vasorelaxation in the rat. Hypertens. Pregnancy. 13:227-244.

30. Yamasaki, M., M.D. Lindheimer, and J.G. Umans. 1996. Effects of pregnancy on femoral microvascular responses in the rat. Am. J. Obstet. Gynecol. 175:730-736.

31. Cockell, A.P., and L. Poston. 1996. Isolated mesenteric arteries from pregnant rats show enhanced flow-mediated relaxation but normal myogenic tone. J. Physiol. 495:545-551.

32. Cockell, A.P., and L. Poston. 1997. 17 ß-oestradiol-induced enhanced flow mediated relaxation in small mesenteric arteries from prepubertal female rats. Hypertens. Pregnancy. 16:72a. (Abstr.)

33. Cockell, A.P., and L. Poston. 1997. Flow-mediated vasodilation is enhanced in normal pregnancy but reduced in preeclampsia. Hypertension. 30: 247-251.

34. Knock, G.A., and L. Poston. 1996. Bradykinin-mediated relaxation of isolated maternal resistance arteries in normal pregnancy and preeclampsia. Am. J. Obstet. Gynecol. 175:1668-1674.

35. Cockell, A.P., J.G. Learmont, A.K. Smárason, C.W.G. Redman, I.L. Sargent, and L. Poston. 1997. Human placental syncytiotrophoblast microvillus membranes impair maternal vascular endothelial function. Br. J. Obstet. Gynecol. 104:235-240.

36. Cameron, N.E., and M.A. Cotter. 1992. Impaired contraction and relaxation in aorta from streptozotocin-diabetic rats: role of polyol pathway. Diabetologia. 35:1011-1019.

37. Tesfamariam, B., M.L. Brown, and R.A. Cohen. 1991. Elevated glucose impairs endothelium-dependent relaxation by activating protein kinase C. $J$. Clin. Invest. 87:1643-1648.

38. Taylor, P.D., J.E. Graves, and L. Poston. 1995. Selective impairment of acetylcholine-mediated endothelium-dependent relaxation in isolated resistance arteries of the streptozotocin-induced diabetic rat. Clin. Sci. 88:519-524.

39. Watson, S.P., and E.G. Lapetina. 1985. 1,2-Diacylglycerol and phorbol ester inhibit agonist-induced formation of inositol phosphates in human platelets: possible implications for negative feedback regulation of inositol phospholipid hydrolysis. Proc. Natl. Acad. Sci. USA. 82:2623-2626.

40. Shimokawa, H., N.A. Flavahan, and P.M. Vanhoutte. 1991. Loss of endothelial pertussis toxin-sensitive $\mathrm{G}$ protein function in atherosclerotic porcine coronary arteries. Circulation. 83:652-660.

41. Gilligan, D.M., V. Guetta, J.A. Panza, C.E. Garcia, A.A. Quyyumi, and R.O. Cannon, III. 1994. Selective loss of microvascular endothelial function in human hypercholesterolemia. Circulation. 90:35-41.

42. Liao, J.K., and C.J. Homcy. 1993. The G proteins of the $\mathrm{G} \alpha_{\mathrm{i}}$ and $\mathrm{G} \alpha_{\mathrm{q}}$ family couple the bradykinin receptor to the release of endothelium-derived relaxing factor. J. Clin. Invest. 92:2168-2172. 